\title{
Single-Electrode Electrochemistry: Chemically Engineering Surface Adhesion and Hardness to Maximize Redox Work Extracted from Tribocharged Silicon
}

Jinyang Zhang, Stuart Ferrie, Song Zhang, Yan B. Vogel, Chandramalika. R. Peiris, Nadim Darwish, and Simone Ciampi*

School of Molecular and Life Sciences, Curtin Institute of Functional Molecules and Interfaces, Curtin University, Bentley, Western Australia 6102, Australia

*E-mail: simone.ciampi@curtin.edu.au

Keywords: single electrode electrochemistry, tribocharging, contact electrification, surface adhesion and hardness, amorphous silicon, surface chemistry

ABSTRACT: Recent research has demonstrated that heterogeneous charge-transfer reactions are not restricted to conductors, and that electrochemical reactions can occur on the surface of statically charged insulators. However, the exact mechanism by which insulators gain and lose electrical charges remains controversial. Herein we have studied quantitatively the reduction of silver ions on intrinsic amorphous silicon surfaces that are statically charged by contact against plastic polymers. We have quantified the magnitude of the redox work done by the tribocharged silicon surface as a function of its adhesion and hardness, with these two variables being tuned using covalent $\mathrm{Si}-\mathrm{C}$ monolayer chemistries. We observed that metallic particles grow preferentially over surfaces that are relatively soft (low DMT modulus) and highly adhesive, hence indirectly proving that the triboelectrification of an insulator-insulator dynamic contact is caused by the exchange of ionic fragments, rather than by the movement of free electrons. This work clarifies on the origin of triboelectricity, devises a surface-chemistry 
method to maximize tribocharging with immediate scope in single-electrode electrochemistry, and describes a concept potentially suitable for the mask-free and bias-free patterning of metal nanoparticles on photoconductors.

\section{INTRODUCTION}

The contact and separation of two insulators generates electrical charges, and from the transfer of toner inks to preventing dangerous electrical discharges, ${ }^{1,2}$ the phenomenon of static electrification holds considerable importance in engineering and technology. Ordered material triboelectric series have been compiled to help predicting charging magnitude and sign, ${ }^{3,4}$ but the exact mechanism of the triboelectric effect is still controversial. ${ }^{5-7}$ How mechanically rubbed insulators become electrified - whether this involves electrons or adsorbed ions -

remains debated. ${ }^{5,6,8,9}$ In a recent work we have revealed that heterogeneous redox reactions on the surface of tribocharged polymers are governed by the relative stability of cationic and anionic oligomeric polymer units, rather than by the net charging magnitude of the sample. ${ }^{10}$ Anionic fragments generated under friction on polyvinyl chloride (PVC), polydimethylsiloxane (PDMS), polytetrafluoroethylene (PTFE), and nylon are the source of electrons accounting for the heterogeneous reduction of soluble metallic ions to elemental metallic particles. ${ }^{10}$ However, contact electrification involves not only on the electronic properties of the contacting materials, ${ }^{10}$ but also their elastic ${ }^{11-14}$ and topographical ${ }^{15}$ characteristics. Notably, material properties such as surface adhesion ${ }^{16}$ and hardness are rarely accounted for when trying to maximize (or minimize) the electrification of non-conductors.

Here we quantify the magnitude of metal (silver) deposition on electrostatically charged amorphous (a-Si) silicon samples that are covalently modified with organic monolayers of $\alpha, \omega$ diynes to alter surface hardness (Derjaguin-Muller-Toporov, DMT, modulus) and adhesion of the substrate. By charging a-Si electrodes against polyvinyl chloride (PVC), and using them in 
single-electrode electrochemistry experiments, we have studied quantitatively the links between contact electrification and material transfer on this model photoconducting surface. The choice of charging a-Si using PVC rests upon the benefit of the polymer large electron affinity and relatively low ionization energy, the combination of these two factors resulting in charged PVC fragments mediating electrochemical work to a large extent compared to other plastics. ${ }^{10}$ The net electrical charge of the samples was measured by Faraday pail readings, while X-ray photoelectron spectroscopy (XPS), atomic force microscopy (AFM), and scanning electron microscopy (SEM) were used to gauge the magnitude of material transfer and of redox work delivered from the charged insulator to a solution of metallic ions.

\section{RESULTS AND DISCUSSION}

The overall process - the reductive nucleation of silver nanoparticles on tribocharged amorphous silicon - is depicted in Scheme 1. Faraday pail data in Figure 1a indicate that the net static charging of amorphous silicon (a-Si) samples tribocharged against PVC (the experimental set-up is illustrated in Figure S1, Supporting Information) develops gradually as a function of the contact time, and these charges persist several minutes after separation (Figure S2, Supporting Information). Silicon samples used in this work are coated with a $4 \mu \mathrm{m}$-thick photoconductive a-Si layer, ${ }^{17-19}$ which is un-doped and therefore highly resistive when shielded from direct light (Figure S3, Supporting Information). All the tribocharging procedures and discharge experiments reported here are therefore obtained on dark samples and at room temperature. Silicon substrates, unlike plastic samples, can be chemically modified using established monolayer chemistries. Furthermore, hydrogen-terminated a-Si retains a surface

reactivity comparable to that of crystal silicon surfaces. ${ }^{17,19}$ This is important, as conventional semiconducting crystalline silicon wafers, ${ }^{20}$ even when poorly doped, were found to acquire less charge than a-Si, to dissipate it very rapidly (Figure S4, Supporting Information), and were 
therefore unable to mediate redox work to an appreciable extent (see XPS and SEM images in Figure S5, Supporting Information). Furthermore, compared to crystalline silicon, a key additional benefit of the a-Si photoconductive layer is that of its light-assisted ON switch of conductance. ${ }^{18,19}$ Regardless of the direction of an external bias, the a-Si layer is a perfect insulator in the dark, while when illuminated it acts as a near-reversible electrode, allowing us to access measurements of monolayer surface coverages by electrochemical means (vide infra). Regarding the surface modification of a-Si samples with 1,6-heptadiyne and 1,8-nonadiyne, different UV-exposure times were tested to ensure comparable surface coverages of the two molecules. The monolayer coverage was indirectly inferred by means of reacting the acetyleneterminated monolayer with azidomethylferrocene (Supporting Information, Scheme S1) ${ }^{21}$ and then resorting to charge measurements in cyclic voltammetry $(\mathrm{CV})^{22}$ to compare surface coverages of the metallocene (Supporting Information, Figure S6). CV curves in Figure S6c indicate that when the hydrosilylation reaction time is set to $40 \mathrm{~min}$ for 1,8 -nonadiyne and to 2 $\mathrm{h}$ for 1,6-heptadiyne, the ferrocene surface coverages reache similar values (on a-Si modified with 1 ,6-heptadiyne is $6.53 \times 10^{-11} \mathrm{~mol} \mathrm{~cm}^{-2}$, and on 1 ,8-nonadiyne surfaces is $6.62 \times 10^{-11}$ mol $\mathrm{cm}^{-2}$ ). These two hydrosilylation reaction times were then used throughout this work.

Our Faraday pail measurements indicate that contact between a-Si (native or chemically modified, Figure 1a) and PVC always results in silicon gaining a net positive charge, and that this charge scales almost linearly with contact time. Furthermore, the charging magnitude is evidently larger in the monolayer-modified samples. PVC has a relatively low ionization energy (stable cations) and give rise to relatively unstable anions (large negative electron affinity). The first hypothesis is therefore that of a link between tribocharging and material transfer - as opposed to electron $\operatorname{transfer}^{8}$ - with the positive charge on the silicon indicating a transfer of fragments from PVC to silicon. Notably, surface water is known to play a crucial role in the electrification of dielectrics, ${ }^{4,23-25}$ and acetylene-terminated monolayers have a great 
ability to rapidly adsorb water. ${ }^{26}$ Measurements of the static charges developed on a-Si samples (with and without monolayers) rubbed against PVC inside a glove box, under argon atmosphere with water levels below $0.3 \mathrm{ppm}$, showed consistently lower Coulombs readings than in air (Figure S7, Supporting Information).

The parallel between the Faraday pail data in Figure 1a and the linear growth with contact time of the $\mathrm{Cl} 2 \mathrm{p}$ XPS emission for all silicon samples, chemically modified or not (Figure $1 \mathrm{~b}-\mathrm{d}$ ), reinforces the conclusion that charged fragments exchanged between PVC and a-Si are the reason for the photoconductor becoming charged. The rate of material transfer inferred from the chlorine-related XPS signal at $198 \mathrm{eV}$ is lower in native a-Si than in samples modified with an organic layer, which is again consistent with the Faraday pail results in Figure 1a. The larger material transfer and larger static electrification observed for samples modified with an aliphatic monolayer could potentially indicate a new means of guiding the electrification of dielectrics, and consequently the extent to which a stand-alone, un-biased, tribocharged insulator can deliver redox work. We therefore attempted to gain further quantitative insights on the links between the surface chemical and physical properties and the extent to which charged plastic fragments are transferred to it. One obvious possibility is that the transfer of plastic fragments towards the a-Si surface is a function of softness, ${ }^{27}$ and adhesion. We used atomic force microscopy (AFM, Figure 2) to probe surface adhesion and hardness and found that a-Si samples modified with either 1,6-heptadiyne or 1,8-nonadiyne are significantly softer (with Young's modulus, according to the Derjaguin-Muller-Toporov model, ${ }^{11}$ of 4.40 and 4.52 GPa respectively) and more adhesive (surface adhesion of 15.5 and $12.1 \mathrm{nN}$, respectively) than native a-Si (DMT modulus of $18.2 \mathrm{GPa}$ and adhesion of $5.54 \mathrm{nN}$ ). The DMT modulus and adhesion are quantitatively determined with respect to standard samples of polystyrene and low-density polyethylene (PS/LDPE, left panels in Figure 2). 
Guided by previous work describing the heterogeneous reduction of metallic ions into metallic nanoparticles on tribocharged plastics, ${ }^{10}$ we attempted electrodeposition experiments on a-Si samples charged against PVC. Tribocharged a-Si samples were immersed in aqueous solutions of $\mathrm{AgNO}_{3}$ and their XPS analysis consistently showed two $\mathrm{Ag} 3 \mathrm{~d}$ spin-orbit-split emissions (Figure 3, $\mathrm{Ag} 3 \mathrm{~d}_{3 / 2}$ and $\mathrm{Ag} 3 \mathrm{~d}_{5 / 2}$ at $374.4 \mathrm{eV}$ and $368.4 \mathrm{eV}$, respectively) of spectral position consistent with literature values for metallic silver. ${ }^{28,29}$ Adventitious shifts to binding energies were accounted for by means of applying a rigid shift to bring the $\mathrm{C} 1 \mathrm{~s} \mathrm{C}-\mathrm{C}$ band to 284.8 $\mathrm{eV} .{ }^{30}$ Increasing to the contact time (i.e. charging magnitude, Figure 1a) increases the amount of charged PVC fragments transferred to the silicon (Figure 1b-d), and in turn it maximizes the extent to which this tribocharged insulating surface can assist heterogeneous redox reactions (Figure 3). XPS data of the silver region demonstrate this point (Figure 3a), which is further corroborated by SEM images in Figure $3 b$.

It is therefore evident that fine-tuning of the triboelectrification of an insulator-insulator dynamic contact can be altered by modifying the DMT modulus and adhesion of the receiving surface. Surfaces of a-Si that are modified with films derived from 1,6-heptadiyne have the lowest DMT modulus and highest adhesion (Figure 2), and this appears to facilitate the transfer of charged fragments from a PVC surface. Overall these fragments bear a net positive charge, but due to the "mosaic" nature of triboelectricity, ${ }^{5,31-33}$ despite anions being out-balanced by cations, ${ }^{10}$ the former are the effector of the reductive electrochemical work done by the a-Si electrode. In line with this, as revealed by the XPS and SEM data in Figure 3b, the magnitude of silver deposition scales directly with surface adhesion but inversely with surface hardness. The situation for the a-Si modified with 1,8-nonadiyne, despite a marginally lower adhesion, is similar to that of 1,6-heptadiyne-modified samples (Figure 3), while hard and poorly adhesive surfaces, such as the native a-Si surface, were found to be decisively less effective in converting mechanical work (friction) into electrochemical work (Figure 3a). 
Our proof-of-principle data also show that there are differences in the density $\left(0.45 / \mu \mathrm{m}^{2}\right.$, $15.8 / \mu \mathrm{m}^{2}$ and $12.9 / \mu \mathrm{m}^{2}$ for a-Si, a-Si with 1,6-heptadyine and 1,8-nonadyine) and mean size $\left(5.2 \times 10^{3}, 4.5 \times 10^{3}\right.$ and $3.6 \times 10^{3} \mathrm{~nm}^{2}$ for a-Si, a-Si with 1,6 -heptadyine and 1,8-nonadyine) of the metal particles that nucleate on a tribocharged surface (Figure 3b). This is possibly associated to differences in surface adhesion, with the higher adhesion leading to an higher surface density of particles (Figure 4). The sequence of building-up static charges on a-Si (contact-separation against a polymer surface), followed by their discharge to reduce ions into metallic particles, could in principle lay the foundation for a new type of mask-free and external bias-free electrochemical lithography. To demonstrate this point we rolled along a straight line on the a-Si samples, and at a constant speed of $0.45 \mathrm{~mm} / \mathrm{s}$, spheres made of PVC and PTFE (Figure 5a). The force ${ }^{34}$ applied to the sphere is maintained constant by means of transferring force through a spring (the set-up is described in Figure S8 of the Supporting Information). Particles were deposited by discharging silver ions on the charged a-Si, and the SEM images in Figure 5a show that metallic line-shaped patterns match the trajectory along which the plastic PVC or PTFE spheres were moved along the a-Si surface. Furthermore, it appears that the particle density and size relates to the polymer mechanical properties (Supporting Information, Figure S9). In other words, it depends on the relative adhesion and softness of at least one of the contacting materials. In the a-Si/PTFE and the a-Si/PVC pairs, PVC is significantly softer and of higher adhesion (DMT modulus of $0.95 \mathrm{GPa}$ and adhesion of $29.4 \mathrm{nN}$, as measured by AFM PeakForce ${ }^{\mathrm{TM}} \mathrm{QNM}^{\mathrm{TM}}$, Figure S9, Supporting Information) than PTFE (4.61 GPa and 2.0 $\mathrm{nN}$ ). Consequently, there is a larger material transfer to a-Si from PVC than from PTFE, which leads to more densely distributed metallic particles on the a-Si sample.

\section{CONCLUSIONS}


This work explores the electrochemical reactivity of statically charged insulators. We reveal a relationship between the amount of charged material that is transferred in a dynamic insulator-insulator contact and the amount of redox work delivered when the tribocharged insulator is discharged in a solution of reducible species. Using established $\mathrm{Si}-\mathrm{C}$ monolayer chemistries we have systematically varied the surface hardness and adhesion of an intrinsic amorphous silicon surface, and using Faraday pail, AFM, SEM and XPS measurements we have quantified the growth of silver particles on tribocharged silicon and observed that these grow preferentially over surfaces that are relatively soft (low DMT modulus) and highly adhesive. These findings extend our understanding of static electricity, devise a surfacechemistry method to maximize tribocharging in a single-electrode electrochemistry set-up, ${ }^{8}$ and outline a new concept suitable for the mask-free and bias-free patterning of metal nanoparticles on insulators or photoconductors. We also believe that the contact electrification of dielectric particles in vigorously agitated suspensions may find application in the emerging field of electrostatic catalysis, ${ }^{35-37}$ perhaps where a vortexed ${ }^{38,39}$ suspension of insulating particles can guide this form of catalysis beyond diffusion limits. This knowledge will also provide the basis to develop devices harvesting electricity from vibrations and friction, akin to the tribo-tunneling current generation reported by metal-insulator semiconductor triboelectric contacts..$^{40-42}$

\section{EXPERIMENTAL SECTION}

Chemicals and Materials. Redistilled solvents and Milli- $\mathrm{Q}^{\mathrm{TM}}$ water $(>18 \mathrm{M} \Omega \mathrm{cm})$ were used for substrate cleaning and to prepare electrolytic solutions. Chemicals used in surface modification procedures and electrochemical experiments were of high purity (>99\%). Hydrogen peroxide (30 wt $\%$ in water), ammonium fluoride (Puranal ${ }^{\mathrm{TM}}, 40 \mathrm{wt} \%$ in water), and sulfuric acid (Puranal $\left.{ }^{\mathrm{TM}}, 95-97 \%\right)$ used in wafer cleaning and etching procedures were of semiconductor grade and obtained from Sigma-Aldrich. 1,8-Nonadiyne (Sigma-Aldrich, 98\%) 
and 1,6-heptadiyne (Sigma-Aldrich, 97\%) were used as received. Sheets of polyvinyl chloride approximately $0.3 \mathrm{~cm}$ thick (PVC, McMaster-Carr, CAT\# 87545K521) were cut into squares of $1 \times 1 \mathrm{~cm}$. PVC and PTFE spheres (4.76 and $1.59 \mathrm{~mm}$ in diameter, respectively) were purchased from Miniature Bearings Australia Pty Ltd. The procedure followed for the deposition of a $4 \mu \mathrm{m}$ thick layer of intrinsic amorphous silicon film on $500 \pm 25 \mu \mathrm{m}$ thick, $\mathrm{n}$ type, $0.007-0.013 \Omega \mathrm{cm}, \mathrm{Si}(100)$ is reported elsewhere. ${ }^{17}$ The dark resistivity of the a-Si film was $10^{4} \Omega \cdot \mathrm{cm}$. Prime grade, single-side polished $\mathrm{Si}(100)$ wafers, n-type, $500 \pm 25 \mu \mathrm{m}$ thick, and with nominal resistivity of $8-12 \mathrm{ohm} \mathrm{cm}$, were obtained from Siltronix, S.A.S. (Archamps, France).

Contact electrification. Prior to the contact charging procedure, PVC, PTFE and a-Si samples were washed with water, methanol, dichloromethane and then dried under a nitrogen flow. Unless stated otherwise, experiments were performed under ambient conditions $\left(23^{\circ} \mathrm{C}\right.$ and $\mathrm{RH}$ of ca. 58\%). Charges on the a-Si samples were measured using a Faraday cup connected to an electrometer (JCI 140 static monitor and JCI 147 charge measurement units, both purchased and calibrated by DEKRA, Southampton Science Park, Southampton, UK), and operating on the $10^{-9}$ Coulomb scale. PVC sheets were fixed on the lower end of a rapidly rotating mechanical shaft (ca. $90 \mathrm{rpm})$ and held against the a-Si surface by means of a spring (spring constant of $4.8 \mathrm{~N} / \mathrm{m}$ ) pressing the back side of the silicon to ensure reproducible contact force (the experimental set-up is illustrated in Figure S1a, Supporting Information). The duration of the contact charging was varied between 5 and 30 min, after which the a-Si's net charge was immediately measured in the Faraday cup. Charging data are reported as charge density (charge-to-geometric area ratios). The motorized apparatus for the movement of plastic spheres on the a-Si surface is detailed in Figure S8, Supporting Information. When specified, tribocharging experiments and Faraday pail measurements were conducted inside a glove box 
(Innovative Technology, PL-HE-2GB fitted with PL-HE-GP1 inert gas purifier) with water and oxygen levels below $0.3 \mathrm{ppm}$.

Surface modification and characterization. Samples of a-Si were cut into $1 \times 1 \mathrm{~cm}$ squares, cleaned with dichloromethane, isopropyl alcohol and water, then immersed for $20 \mathrm{~min}$ in a hot piranha solution $\left(130{ }^{\circ} \mathrm{C}, 3: 1(\mathrm{v} / \mathrm{v})\right.$ mixture of concentrated sulfuric acid and $30 \%$ hydrogen peroxide). Samples were then rinsed thoroughly with water before being etched for $10 \mathrm{~min}$ in $40 \%$ aqueous ammonium fluoride that was degassed by bubbling argon gas for $20 \mathrm{~min}$. A small amount of ammonium sulfite was added to the etching solution as oxygen scavenger. The hydrogen-terminated samples were then rinsed sequentially with water and dichloromethane, dried under a nitrogen stream, and then placed on a glass microscope slide. A small sample (ca. $50 \mu \mathrm{L}$ ) of either 1,8-nonadiyne or 1,6-hepdiyne was then dropped on the wafer. The UVassisted (Vilber,VL-215.M, $\lambda=312 \mathrm{~nm}$, nominal power output of $30 \mathrm{~W}$ and positioned approximately $200 \mathrm{~mm}$ away from the silicon sample) grafting of 1,8-nonadiyne and 1,6hepdiyne on the hydrogen-terminated amorphous silicon followed minor modifications of literature procedures (Supporting Information, Scheme 1). ${ }^{43}, 44$ The UV reaction time for the 1,8-nonadiyne grafting was varied between 10 and 40 min (10, 20 and 40 min), while the reaction between 1,6-hepdiyne and a-Si was stopped after either 2,3 or $4 \mathrm{~h}$. To limit its evaporation during the hydrosilylation reaction, the liquid $\alpha, \omega$-diyne sample was top-contacted with a quartz slide. Chemically passivated a-Si samples were then rinsed several times with dichloromethane, rested for $12 \mathrm{~h}$ in a sealed vial under dichloromethane at $+4{ }^{\circ} \mathrm{C}$ and blown dry under a nitrogen stream before being used in the tribocharging and electro-deposition experiments. When a-Si samples were used without an organic monolayer, samples were washed with piranha solution and then immediately tribocharged against PVC.

After being rubbed with PVC or PTFE, tribocharged a-Si samples were transferred to a $50 \mathrm{mM}$ aqueous solution of $\mathrm{AgNO}_{3}$ (99.99\%, Sigma), rested in the liquid for $3 \mathrm{~h}$ under dark, washed 
with water and then dried under a stream of nitrogen. Mechanical properties (DMT modulus and surface adhesion) of both the a-Si and plastic samples were obtained from atomic force microscopy (AFM) measurements using a Bruker Dimension FastScan (Bruker Corporation, Santa Barbara, CA, USA). AFM data were obtained in PeakForce ${ }^{\mathrm{TM}}$ QNM $^{\mathrm{TM}}$ imaging mode, by tapping in air at room temperature and using SNL-10 Bruker probes with a spring constant of $0.350 \mathrm{~N} \mathrm{~m}^{-1}$. The imaging resolution was set to 256 points/line, the scan rate to $2 \mathrm{~Hz}$ and the peak force to $12 \mathrm{nN}$. Standard harmonic samples of polystyrene (PS) and low density polyethylene (LDPE), with discrete domains of 2.1 (PS) and 0.2 GPa (LDPE) moduli, were supplied by Bruker. The values reported for DMT moduli and adhesion are the average values obtained from the analysis of $1 \times 1 \mu \mathrm{m}$ AFM images. The reported DMT moduli are the reduced Young's modulus for the tip-surface contact, not the Young's modulus of the surface. The Young's modulus and Poisson ratio of the tip are assumed to be constant, so that a comparison of moduli between materials is possible. The reported DMT moduli were corrected by applying a rigid shift calculated by bringing the experimental DMT modulus of standard harmonic samples of PS and LDPE to their nominal values. X-ray photoelectron spectroscopy (XPS) surface characterization was performed on a Kratos Axis Ultra DLD spectrometer using a monochromated Al-Ka $(1486.6 \mathrm{eV})$ irradiation source operating at $150 \mathrm{~W}$. Spectra were taken in normal emission at or below $7 \times 10^{-9}$ Torr. Data files were processed using CasaXPS $\odot$ software. Binding energies are corrected by applying a rigid shift to bring the $\mathrm{C}-\mathrm{C} 1 \mathrm{~s}$ emission to $284.8 \mathrm{eV}$. 
Scheme 1. Electrochemical growth of silver nanoparticles on tribocharged amorphous silicon(a-Si) samples. The surface of the a-Si photoconductor was either unmodified or coated with a covalently linked monolayer of 1,6-heptadiyne (shown) or 1,8-nonadiyne. The presence of the organic monolayer is aimed at defining the surface hardness and adhesion of the a-Si surface. Surface adhesion and hardness govern material transfer from PVC to a-Si. PVC fragments mediates the electrochemical reduction of silver ions to metallic silver.
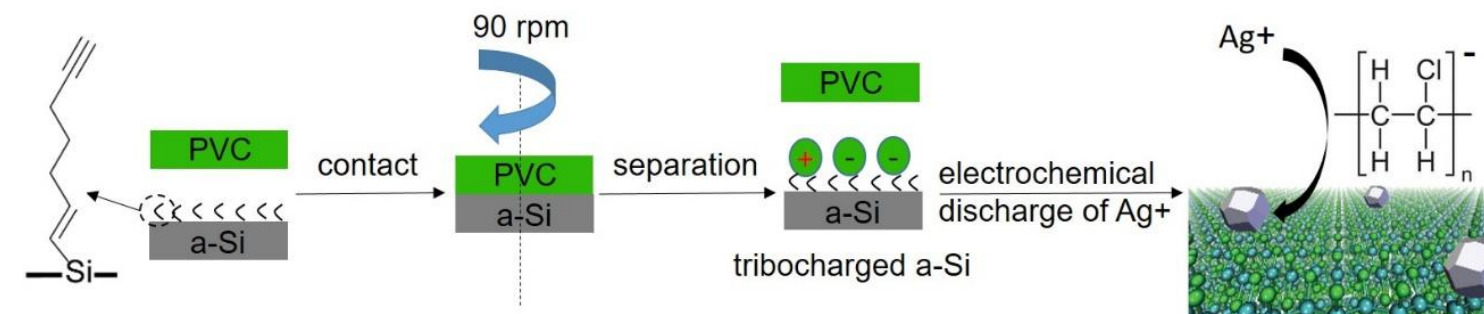

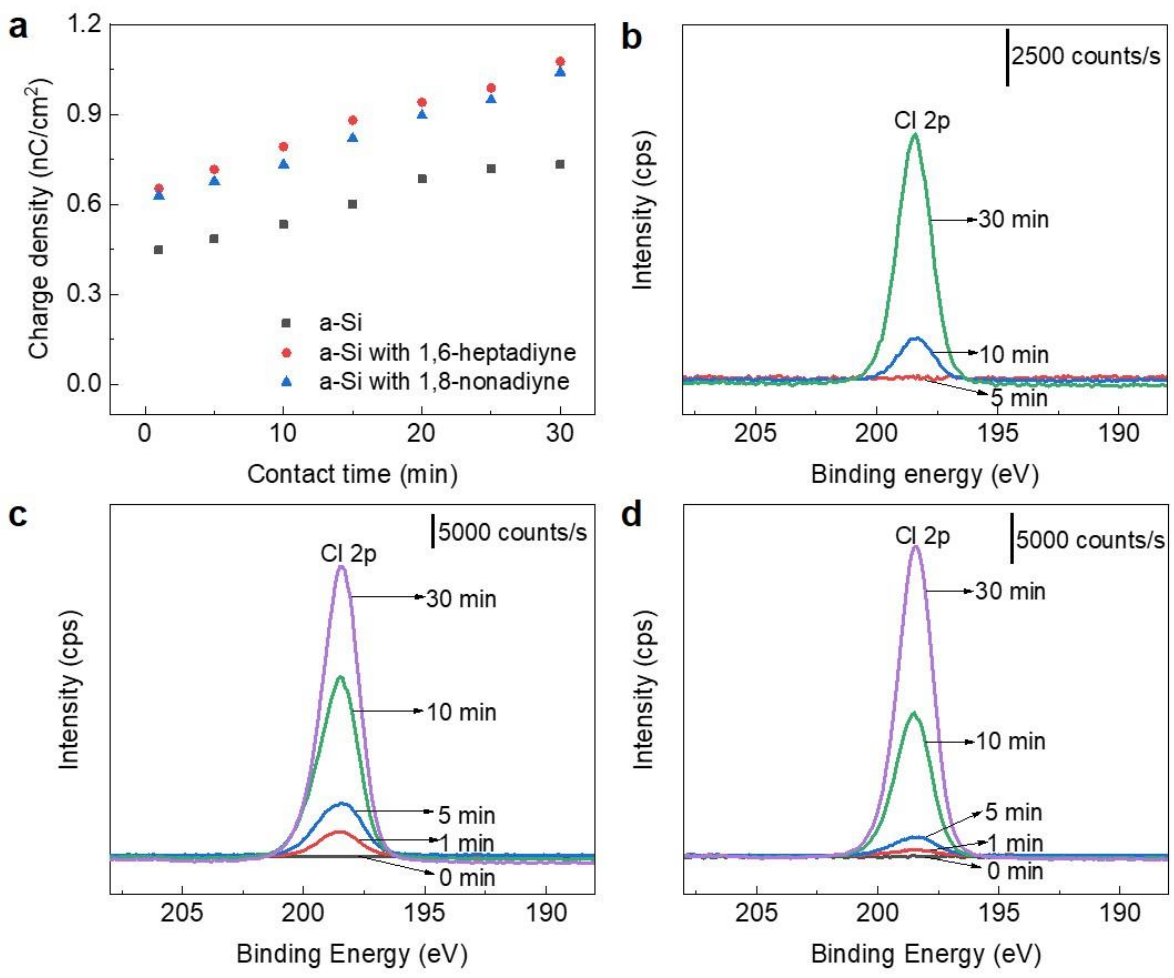

Figure 1. (a) Variation of the charge density (net charge divided by the sample geometric area) of a-Si samples tribocharged against PVC as a function of the contact-charging time. For all substrates, charging increases with contact-charging time. (b-c) High resolution $\mathrm{Cl} 2 \mathrm{p}$ XPS emission of amorphous silicon samples tribocharged against PVC. Data for unmodified a-Si (b), for a-Si modified with 1,6-heptadiyne (c), and for a-Si modified with 1,8-nonadiyne (d). The contact-charging time is specified by labels to the curves. The transfer of polymer fragments to a-Si upon contact is evidenced by a gradual increase in the intensity of the $198 \mathrm{eV} \mathrm{Cl} 2 \mathrm{p}$ emission. 


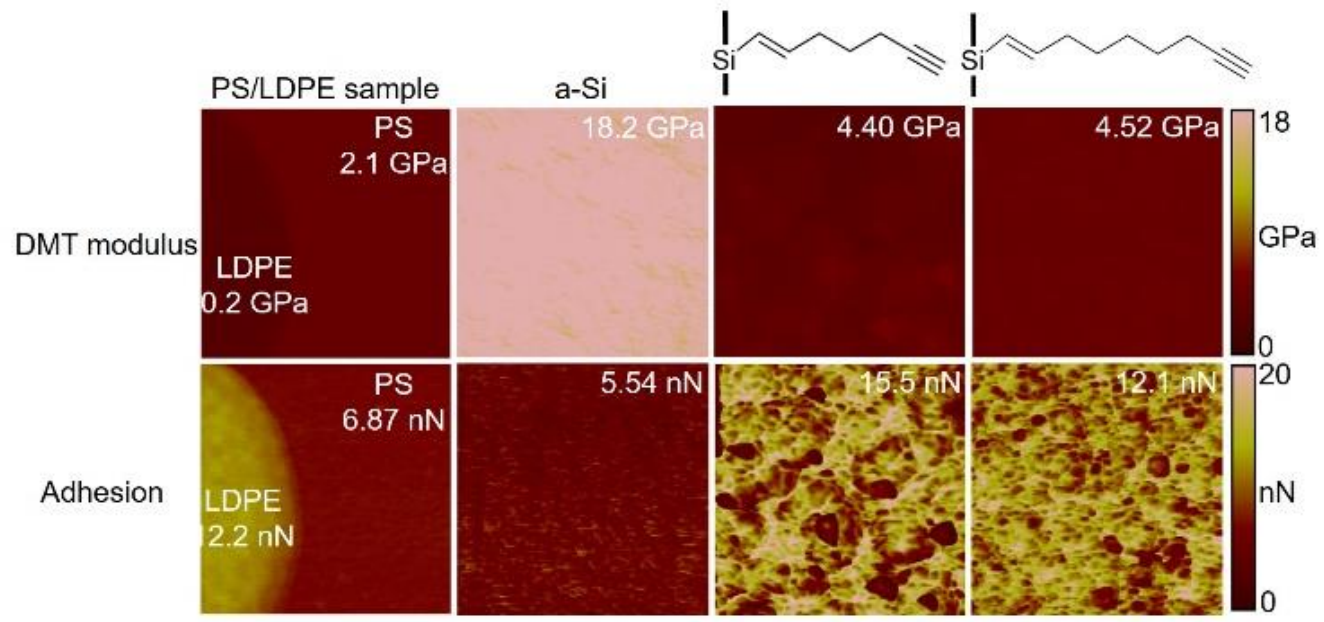

Figure 2. Atomic force microscopy PeakForce ${ }^{\mathrm{TM}} \mathrm{QNM}^{\mathrm{TM}}$ measurements of surface hardness (DMT modulus) and adhesion for standard samples of polystyrene and low density polyethylene (PS/LDPE), native amorphous silicon (a-Si), and chemically-modified a-Si samples. The moduli and adhesion are quantitatively determined with respect to the standard PS/LDPE sample. Imaged areas are $1 \times 1 \mu \mathrm{m}$ for all samples. 

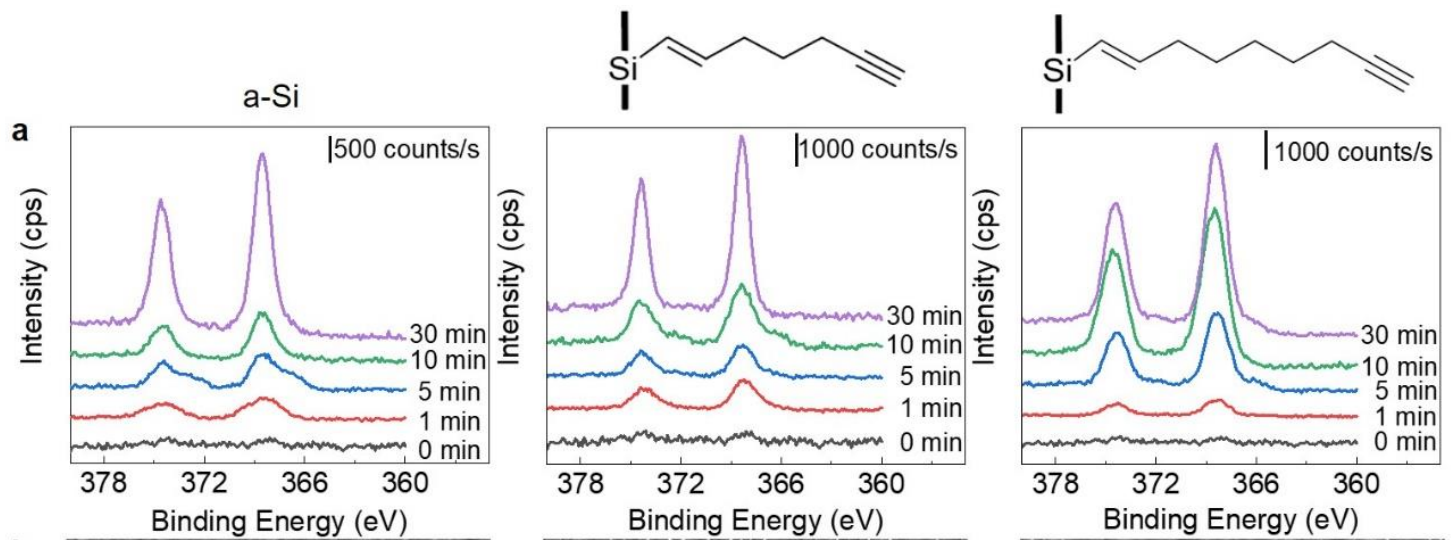

b
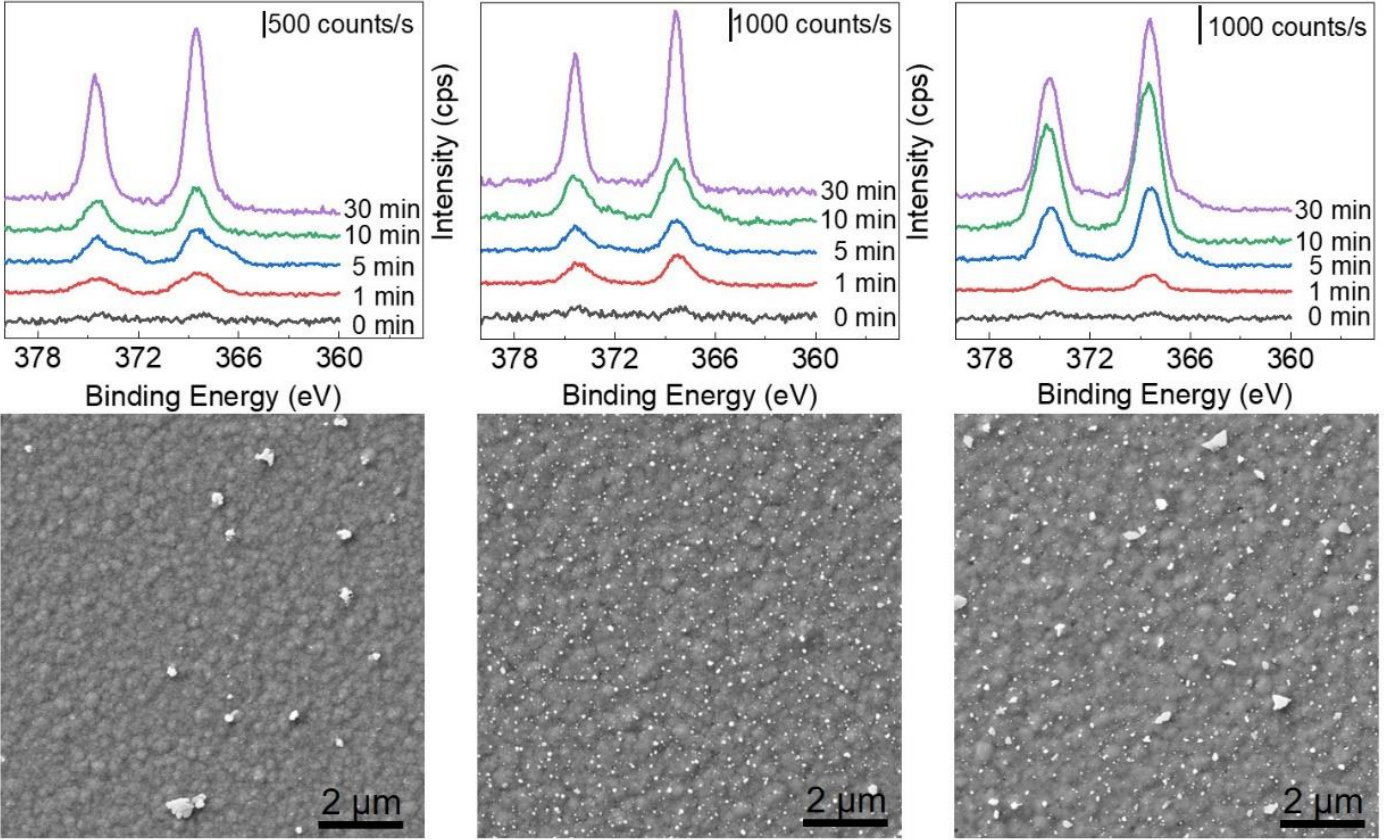

Figure 3. Heterogeneous redox work done by statically charged un-doped a-Si samples. Silver ions are reduced to metallic silver particles by the tribocharged a-Si surface and the extent of the redox work scales with the duration of the contact-charging against a PVC surface. (a) Evolution of the $\mathrm{Ag} 3 \mathrm{~d}$ XPS signal on tribocharged a-Si samples that are discharged after being immersed in a $50 \mathrm{mM}$ aqueous solution of $\mathrm{AgNO}_{3}$. The spectral position of the two spin-orbit-split $\mathrm{Ag} 3 \mathrm{~d}$ emissions $\left(\mathrm{Ag} 3 \mathrm{~d}_{3 / 2}\right.$ and $\mathrm{Ag} 3 \mathrm{~d}_{5 / 2}, 374.4 \mathrm{eV}$ and $368.4 \mathrm{eV}$, respectively) is consistent with literature values for metallic silver. The contact time is specified by labels adjacent to the XPS traces. Curves are vertically offset for clarity and the absolute ordinate has no physical meaning. (b) SEM images of Ag particles deposited on either charged a-Si, a-Si modified with 1,6-heptadiyne and a-Si modified with 1,8-nonadiyne (left to right). 

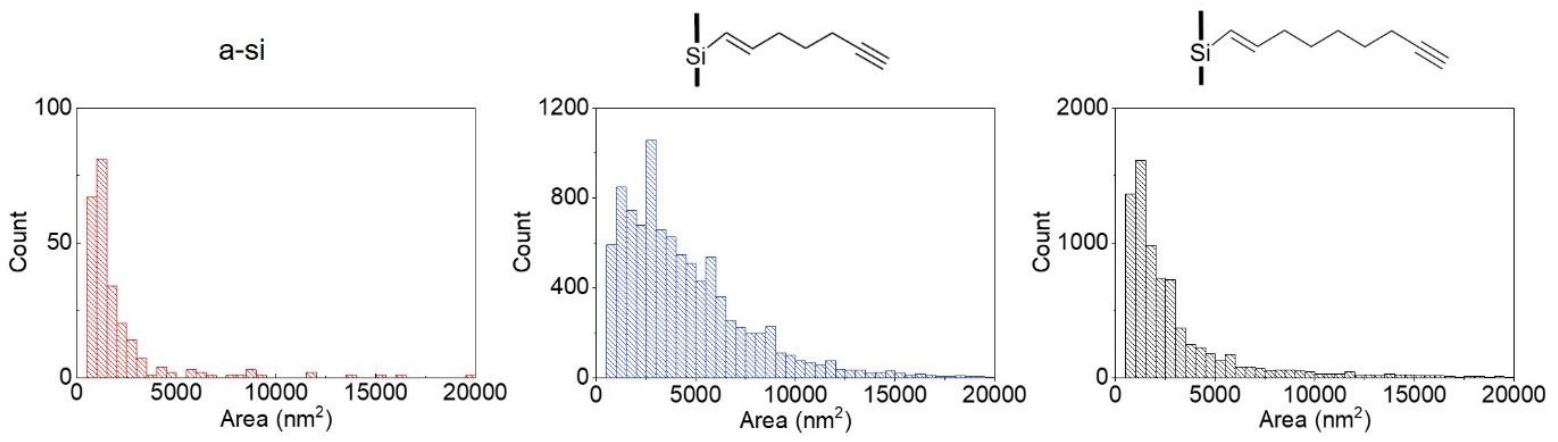

Figure 4. Size distribution of silver particle that are electrochemically grown on samples of a-Si, a-Si modified with 1,6-heptadyine and a-Si modified with 1,8-nonadyine. All samples were first tribocharged against PVC and then discharged in aqueous $50 \mathrm{mM} \mathrm{AgNO}_{3} \mathrm{solutions}$ 

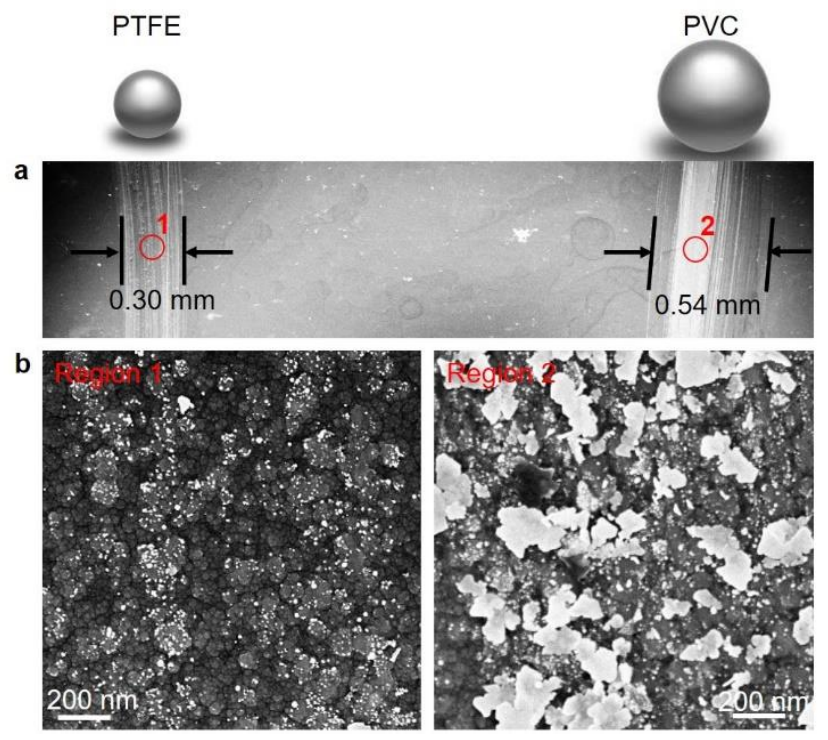

Figure 5. Metallic patterns grown on tribocharged regions of an insulator. (a) SEM images of line-shaped patterns of silver particles grown on a-Si samples tribocharged by rolling on the surface PVC and PTFE spheres. The pattern "develops" after immersion - i.e. discharge - of the tribocharged samples in aqueous solutions of $\mathrm{AgNO}_{3}$. (b) SEM data comparing the density and size of $\mathrm{Ag}$ particles within regions 1 and 2 (red circles marked in panel a). Region 1 (tribocharged with PTFE spheres) and region 2 (PVC) had, respectively, small silver particles with diameter around 20 nm, and larger metallic deposits (around $200 \mathrm{~nm}$ ).

\section{ASSOCIATED CONTENT}

\section{Supporting Information}

The Supporting Information is available free of charge on the ACS Publications website.

Detailed experimental procedures and instrument set-up schemes, SEM images, AFM, XPS and electrochemical data.

\section{Corresponding Author}

simone.ciampi@curtin.edu.au

\section{Author Contributions}

The manuscript was written with contributions by all authors. All authors have approved of the final version of the manuscript. 


\section{Notes}

The authors declare no competing financial interest.

\section{ACKNOWLEDGMENTS}

This work was financially supported by the Australian Research Council (DE160100732 (S.C.), DE160101101 (N.D.), DP190100735 (S.C and N.D.)).

\section{REFERENCES}

(1) Childress, C. O.; Kabell, L. J., Electrostatic printing system. Google Patents: 1963.

(2) Collocott, S.; Morgan, V.; Morrow, R., Electrostatic Hazard Involved in Discharging $\mathrm{CO}_{2}$ Fire Extinguisher. Proc. Inst. Elect. Engrs. 1980, 127, 119-120.

(3) Zhang, J.; Ciampi, S., The Position of Solid Carbon Dioxide in the Triboelectric Series. Aust. J. Chem. 2019, 72, 633-636.

(4) Burgo, T. A.; Galembeck, F.; Pollack, G. H., Where is Water in the Triboelectric Series? J. Electrost. 2016, 80, 30-33.

(5) Baytekin, H.; Patashinski, A.; Branicki, M.; Baytekin, B.; Soh, S.; Grzybowski, B. A., The Mosaic of Surface Charge in Contact Electrification. Science 2011, 333, 308-312.

(6) Liu, C.-Y.; Bard, A. J., Electrostatic Electrochemistry at Insulators. Nat. Mater. 2008, 7, 505509 .

(7) Diaz, A. F.; Guay, J., Contact Charging of Organic Materials: Ion vs. Electron Transfer. IBM J. Res. Dev. 1993, 37, 249-260.

(8) Liu, C.; Bard, A. J., Chemical Redox Reactions Induced by Cryptoelectrons on a PMMA Surface. J. Am. Chem. Soc. 2009, 131, 6397-6401.

(9) Galembeck, F.; Burgo, T. A.; Balestrin, L. B.; Gouveia, R. F.; Silva, C. A.; Galembeck, A., Friction, Tribochemistry and Ttriboelectricity: Recent Progress and Perspectives. RSC Adv. 2014, 4, 64280-64298.

(10) Zhang, J.; Rogers, F.; Darwish, N.; Gonçales, V. R.; Vogel, Y. B.; Wang, F.; Gooding, J. J.; Peiris, M. C.; Jia, G.; Veder, J.-P.; Coote, M. L.; Ciampi, S., Electrochemistry on Tribocharged Polymers is Governed by the Stability of Surface Charges Rather than Charging Magnitude. J. Am. Chem. Soc. 2019, 141, 5863-5870.

(11) Derjaguin, B. V.; Muller, V. M.; Toporov, Y. P., Effect of Contact Deformations on the Adhesion of Particles. J. Colloid Interface Sci. 1975, 53, 314-326.

(12) Maugis, D., Contact, Adhesion and Rupture of Elastic Solids. Springer Science \& Business Media, 2013, Vol. 130.

(13) Santos, L. P.; da Silva, D. S.; Batista, B. C.; Moreira, K. S.; Burgo, T. A.; Galembeck, F., Mechanochemical Transduction and Hygroelectricity in Periodically Stretched Rubber. Polymer 2019, $171,173-179$.

(14) Camara, C. G.; Escobar, J. V.; Hird, J. R.; Putterman, S. J., Correlation between nanosecond $\mathrm{X}$-ray flashes and stick-slip friction in peeling tape. Nature 2008, 455, 1089.

(15) Bowden, F. P.; Tabor, D., The Area of Contact Between Stationary and Moving Surfaces. Proc. Roy. Soc. A 1939, 169, 391-413.

(16) Sow, M.; Widenor, R.; Kumar, A.; Lee, S. W.; Lacks, D. J.; Sankaran, R. M., Strain - Induced Reversal of Charge Transfer in Contact Electrification. Angew. Chem., Int. Ed. 2012, 51, 2695-2697.

(17) Vogel, Y. B.; Zhang, L.; Darwish, N.; Gonçales, V. R.; Le Brun, A.; Gooding, J. J.; Molina, A.; Wallace, G. G.; Coote, M. L.; Gonzalez, J.; Ciampi, S., Reproducible Flaws Unveil Electrostatic Aspects of Semiconductor Electrochemistry. Nat. Commun. 2017, 8, 2066. 
(18) Vogel, Y. B.; Gooding, J. J.; Ciampi, S., Light-Addressable Electrochemistry at Semiconductor Electrodes: Redox Imaging, Mask-Free Lithography and Spatially Resolved Chemical and Biological Sensing. Chem. Soc. Rev. 2019, 48, 3723-3739.

(19) Vogel, Y. B.; Molina, A.; Gonzalez, J.; Ciampi, S., Quantitative Analysis of Cyclic Voltammetry of Redox Monolayers Adsorbed on Semiconductors: Isolating Electrode Kinetics, Lateral Interactions, and Diode Currents. Anal. Chem. 2019, 91, 5929-5937.

(20) Zhang, L.; Vogel, Y. B.; Noble, B. B.; Goncales, V. R.; Darwish, N.; Brun, A. L.; Gooding, J. J.; Wallace, G. G.; Coote, M. L.; Ciampi, S., TEMPO Monolayers on Si(100) Electrodes: Electrostatic Effects by the Electrolyte and Semiconductor Space-Charge on the Electroactivity of a Persistent Radical. J. Am. Chem. Soc. 2016, 138, 9611-9619.

(21) Ciampi, S.; Le Saux, G.; Harper, J. B.; Gooding, J. J., Optimization of Click Chemistry of Ferrocene Derivatives on Acetylene - Functionalized Silicon (100) Surfaces. Electroanalysis 2008, 20, $1513-1519$.

(22) Darwish, N.; Eggers, P. K.; Ciampi, S.; Tong, Y.; Ye, S.; Paddon-Row, M. N.; Gooding, J. J., Probing the Effect of the Solution Environment around Redox-Active Moieties Using Rigid Anthraquinone Terminated Molecular Rulers. J. Am. Chem. Soc. 2012, 134, 18401-18409.

(23) Biegaj, K. W.; Rowland, M. G.; Lukas, T. M.; Heng, J. Y. Y., Surface Chemistry and Humidity in Powder Electrostatics: A Comparative Study Between Tribocharging and Corona Discharge. ACS Omega 2017, 2, 1576-1582.

(24) Harris, I. A.; Lim, M. X.; Jaeger, H. M., Temperature Dependence of Nylon and PTFE Triboelectrification. Phys. Rev. Mater. 2019, 3, 085603-085612.

(25) Galembeck, F.; Burgo, T. A. L., Chemical Electrostatics: New Ideas on Electrostatic Charging: Mechanisms and Consequences. Springer International Publishing AG, 2017.

(26) James, M.; Darwish, T. A.; Ciampi, S.; Sylvester, S. O.; Zhang, Z.; Ng, A.; Gooding, J. J.; Hanley, T. L., Nanoscale Condensation of Water on Self-Assembled Monolayers. Soft Matter 2011, 7, 5309-5318.

(27) Baytekin, H. T.; Baytekin, B.; Incorvati, J. T.; Grzybowski, B. A., Material Transfer and Polarity Reversal in Contact Charging. Angew. Chem., Int. Ed. 2012, 51, 4843-4847.

(28) Jafarkhani, P.; Torkamany, M.; Dadras, S.; Chehrghani, A.; Sabbaghzadeh, J., Necklace-shaped $\mathrm{Au}-\mathrm{Ag}$ nanoalloys: laser-assisted synthesis and nonlinear optical properties. Nanotechnology 2011, 22, 235703.

(29) Romand, M.; Roubin, M.; Deloume, J.-P., X-ray Photoelectron Emission Studies of Mixed Selenides $\mathrm{AgGaSe}_{2}$ and $\mathrm{Ag}_{9} \mathrm{GaSe}_{6}$. J. Solid State Chem. 1978, 25, 59-64.

(30) Reshetenko, T. V.; St-Pierre, J.; Bethune, K. P.; Artyushkova, K.; Rocheleau, R.; Atanassov, P., Multi-Analytical Study of Gas Diffusion Layer PTFE Content Local Variation. ECS Trans. 2013, 50, 591-599.

(31) Nonnenmacher, M.; O'Boyle, M.; Wickramasinghe, H. K., Surface Investigations with a Kelvin Probe Force Microscope. Ultramicroscopy 1992, 42, 268-273.

(32) Galembeck, A.; Costa, C.; Da Silva, M.; Souza, E.; Galembeck, F., Scanning Electric Potential Microscopy Imaging of Polymers: Electrical Charge Distribution in Dielectrics. Polymer 2001, 42, 4845-4851.

(33) Burgo, T. A.; Silva, C. A.; Balestrin, L. B.; Galembeck, F., Friction Coefficient Dependence on Electrostatic Tribocharging. Sci. Rep. 2013, 3, 2384-2391.

(34) da Silveira Balestrin, L. B.; Del Duque, D.; da Silva, D. S.; Galembeck, F., Triboelectricity in Insulating Polymers: Evidence for a Mechanochemical Mechanism. Faraday Discuss. 2014, 170, 369 383.

(35) Ciampi, S.; Darwish, N.; Aitken, H. M.; Díez-Pérez, I.; Coote, M. L., Harnessing Electrostatic Catalysis in Single Molecule, Electrochemical and Chemical Systems: A Rapidly Growing Experimental Tool Box. Chem. Soc. Rev. 2018, 47, 5146-5164.

(36) Shaik, S.; Ramanan, R.; Danovich, D.; Mandal, D., Structure and Reactivity/Selectivity Control by Oriented-External Electric Fields. Chem. Soc. Rev. 2018, 47, 5125-5145.

(37) Shaik, S.; Mandal, D.; Ramanan, R., Oriented Electric Fields as Future Smart Reagents in Chemistry. Nat. Chem. 2016, 8, 1091-1098.

(38) Britton, J.; Dalziel, S. B.; Raston, C. L., The Synthesis of Di-Carboxylate Esters Using Continuous Flow Vortex Fluidics. Green Chem. 2016, 18, 2193-2200. 
(39) Britton, J.; Meneghini, L. M.; Raston, C. L.; Weiss, G. A., Accelerating Enzymatic Catalysis Using Vortex Fluidics. Angew. Chem., Int. Ed. 2016, 55, 11387-11391.

(40) Liu, J.; Cheikh, M. I.; Bao, R.; Peng, H.; Liu, F.; Li, Z.; Jiang, K.; Chen, J.; Thundat, T., TriboTunneling DC Generator with Carbon Aerogel/Silicon Multi - Nanocontacts. Adv. Electron. Mater. 2019, https://doi.org/10.1002/AELM.201900464.

(41) Liu, J.; Jiang, K.; Nguyen, L.; Li, Z.; Thundat, T., Interfacial Friction-Induced Electronic Excitation Mechanism for Tribo-Tunneling Current Generation. Mater. Horiz. 2019, 6, 1020-1026.

(42) Liu, J.; Miao, M.; Jiang, K.; Khan, F.; Goswami, A.; McGee, R.; Li, Z.; Nguyen, L.; Hu, Z.; Lee, J., Sustained Electron Tunneling at Unbiased Metal-Insulator-Semiconductor Triboelectric Contacts. Nano Energy 2018, 48, 320-326.

(43) Huck, L. A.; Buriak, J. M., UV-Initiated Hydrosilylation on Hydrogen-Terminated Silicon (111): Rate Coefficient Increase of Two Orders of Magnitude in the Presence of Aromatic Electron Acceptors. Langmuir 2012, 28, 16285-16293.

(44) Wagner, P.; Nock, S.; Spudich, J. A.; Volkmuth, W. D.; Chu, S.; Cicero, R. L.; Wade, C. P.; Linford, M. R.; Chidsey, C. E., Bioreactive Self-Assembled Monolayers on Hydrogen-Passivated $\mathrm{Si}(111)$ as a New Class of Atomically Flat Substrates for Biological Scanning Probe Microscopy. $J$. Struct. Biol. 1997, 119, 189-201.

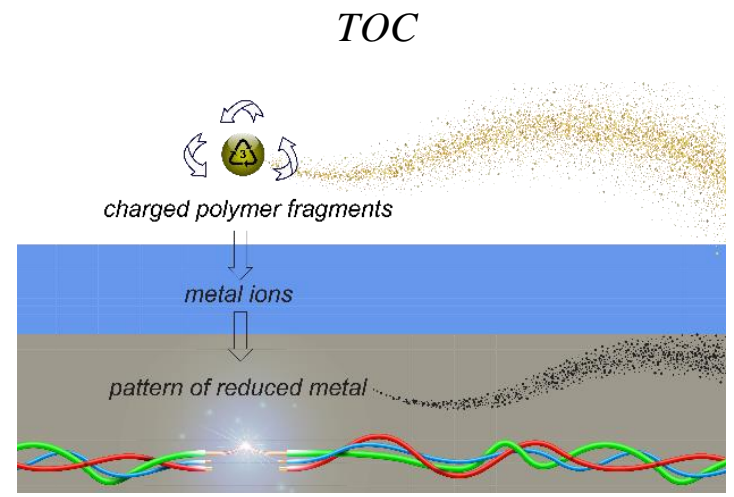

